\title{
Mercadeando causas ambientales
}

\author{
DIANA FORERO RODRÍGUEZ
}

\section{RESUMEN}

En el presente escrito se presentan varias alternativas para el desarrollo del mercadeo de causas ambientales a través de entidades sin ánimo de lucro. En la primera parte se exploran los modelos de mercadeo que se han empelado con mayor regularidad: la filantropía estratégica, el mercadeo social y el mercadeo de causas. Se muestran las actividades más comunes en cada tipo de mercadeo y algunos puntos clave para desarrollarlo. En la segunda parte se presentan las barreras para la gestión del mercadeo de causas ambientales, señalando en qué consisten esas barreras, los puntos críticos y algunas alternativas planteadas desde la comunicación organizacional, la negociación, la gestión de relaciones con clientes y el control de gestión.

\section{PALABRAS CLAVE}

Mercadeo de causas sociales, mercadeo social, filantropía, mercadeo de causas, gestión de mercadeo ambiental.
Las entidades que promueven causas ambientales deben desarrollar una gran cantidad de actividades empresariales, como cualquier otra empresa, entre las que se encuentran las de mercadeo. Las actividades de mercadeo en estas entidades, normalmente sin ánimo de lucro (OSL), están dirigidas principalmente a la consecución de fondos para el desarrollo de los programas que promueva la entidad, a la comunicación y promoción de la causa.

Las actividades de mercadeo pueden dirigirse a personas o a empresas; en este documento nos centraremos en las segundas. El mercadeo dirigido a empresas puede realizarse a través de tres tipos diferentes de modelos estratégicos: el mercadeo basado en la filantropía, el mercadeo social y el mercadeo de causas.

\section{Mercadeo basado en la filantropía estratégica}

En este tipo de mercadeo, uno de los más empleados por las entidades sin ánimo de lucro ${ }^{1}$ (OSL) y por otras organizaciones que promueven causas sociales, consiste en la donación que realizan empresas privadas de una parte de sus fondos o recursos a una causa o a una organización sin ánimo de lucro . Este modelo es lo más cercano a la caridad empresarial. La empresa donante puede aportar dinero, equipos, productos, patrocinar eventos o coordinar el trabajo voluntario de los empleados.

La filantropía no tiene un resultado comercial para la empresa donante. La empresa se beneficia porque permite el desarrollo de alguna estrategia de un área particular que puede estar relacionada con la causa ${ }^{2}$ o con el interés de sus directivos. Un ejemplo se encuentra

1 WYMER, Walter. "Special issue on corporate philanthropy". International Journal of nonprofit and voluntary sector marketing. Vol. 11, No.1, 2006.

2 HOGGINS, Kevin. "Marketing with conscience". Marketing Management.Vol. 11, No.4, 2002. 
en el sector petrolero, en donde se realizan actividades de apoyo a programas ambientales en las comunidades donde se realizan perforaciones para mantener una buena relación con la comunidad y facilitar el proceso de producción de la empresa.

Un programa de mercadeo basado en la filantropía requiere que la entidad sin ánimo de lucro desarrolle un proceso de construcción de imagen alrededor de la entidad y del benefactor. El mercadeo se centra en los principios de la segmentación para la selección de los benefactores y luego, en un proceso de relaciones públicas y venta directa a las entidades benefactoras potenciales. El objetivo del programa de mercadeo debe ser alinear los objetivos de la entidad con los del benefactor o, por lo menos, mostrar el camino para la construcción de esta identidad. Allí se integra la estrategia de imagen de la causa hacia la comunidad con la estrategia de mercadeo dirigida al benefactor.

Como señalan Daellenbach, Davies y Ashill, se deben considerar algunos criterios al establecer una relación de patrocinio; entre ellos se encuentran la visualización de las características del patrocinio en la selección del benefactor ${ }^{3}$, los efectos en la reputación, credibilidad del o los aliados estratégicos, las expectativas de la industria y las normas sociales alrededor de la causa y, lo más importante, el impacto en las redes sociales o en el público objetivo del benefactor. Aunque se apela a la

\section{Diana Forero Rodríguez:}

Psicóloga, especialista en Estadística y candidata a Master en Dirección de Marketing y a Doctora en Ciencias de Gestión. Ha realizado investigaciones en el campo social y del mercadeo, así como múltiples proyectos con empresas privadas y públicas en la gestión de la comunicación, la estrategia de mercadeo y la medición. Actualmente se encuentra vinculada al Politécnico Grancolombiano como Directora de Investigación y Proyección Social y es gerente de su empresa de investigación. Su correo es: dforero@poligran.edu.co filantropía, el proceso de patrocinio y donación tiene al menos objetivos sociales y de relaciones que son de importancia para la empresa benefactora.

\section{Mercadeo Social}

El mercadeo social se ha trabajado como un sistema para analizar el cambio social. Según Kotler, el "marketing social es un proceso para influenciar las ideas y conductas sociales basado en la premisa de que las causas sociales pueden ser mercadeadas de manera semejante a cualquier producto" 4 . Esta especialidad del mercadeo ha sido útil en la promoción de conductas socialmente deseables tales como, la planeación familiar y la prevención del sida, el reciclaje, el uso de los cinturones de seguridad ${ }^{5}$. También se ha empleado en la influencia de movimientos sociales tales como la protección del medio ambiente o la adopción de comportamientos preventivos frente a las incidencias de los cambios ambientales en la salud.

El desarrollo del mercadeo social en las entidades sin ánimo de lucro tiene características semejantes al de las entidades públicas, aunque varían algunos elementos de gestión. En ambos casos se realiza una segmentación; sin embargo ésta tiene dos vertientes: los benefactores y los beneficiaros. La segmentación de los benefactores se realiza en función de los objetivos de la campaña de mercadeo, mientras que la de los beneficiarios consiste en establecer las características del segmento objetivo en su comportamiento frente a la problemática o causa que se está abordando; por ejemplo, tomemos el tema de la contaminación del agua generada por el mal manejo de desechos humanos, animales y de materiales. En este caso, la segmentación del beneficiario consiste en establecer las zonas de mayor contaminación, clasificar las familias en grupos según sus características de comportamiento frente al manejo de desechos o usos del agua y definir cuáles serían los beneficiarios y en qué niveles, para los programas que se adelantarán.

Un segundo aspecto del mercadeo social, señalado por Fox y Kotler y que marca una diferencia importante frente a la filantropía y al mercadeo de causas, es el análisis y el diseño del producto o servicio que se espera que adopte la comunidad para contrarrestar el com-

3 DAELLENBACH, Kate, Davies, Jhon y Ashill, Nicholas. "Understanding sponsorship relationships-multiple frames and multiple perspectives". International Journal of nonprofit and voluntary sector marketing. Vol. 11, No. 1, 2006.

4 FOX, Karen y Kotler, Philip. "The marketing of social causes: The first 10 years". Journal of Marketing. Vol. 44, №. 4, 1980.

5 Holdford, David. "Understanding the dynamics of the pharmaceutical market using a social marketing framework". The journal of monsumer marketing. Vol. 22, No. 7, 2005 
portamiento inadecuado. En el ejemplo citado, puede requerirse, a corto plazo, la creación de un producto que purifique el agua y a mediano plazo, un producto biológico que contrarreste la contaminación, además de productos de almacenaje de desechos. El análisis de los productos o servicios requeridos por el beneficiario da las posibilidades para enlazar la causa con empresas y Estado u otras entidades benefactoras.

El tercer aspecto del mercadeo social es el desarrollo de incentivos ${ }^{6}$. En este caso implica las actividades de comunicación y promoción hacia los beneficiarios y benefactores para vincularse a la causa y promoverla activamente, adoptando los comportamientos de interés. Allí aparecen las actividades de relaciones públicas como patrocinios, eventos u otras actividades que incentiven la adherencia a la causa o a los programas desarrollados por la entidad.

El cuarto aspecto en el programa de mercadeo social es la facilitación ${ }^{7}$, allí la función del mercadeo es la de crear canales de respuesta que disminuyan los costos y esfuerzos tanto de beneficiarios como de benefactores. Esto puede implicar buscar alternativas con proveedores de insumos, canales de distribución, medios de comunicación o entidades gubernamentales. El objetivo es relacionar a las personas y a las empresas con los comportamientos que quieran que sean adoptados a favor de la causa.

La gestión de mercadeo en este caso también busca organizar una planeación en la que se realizan esfuerzos por buscar la máxima satisfacción de beneficiarios y benefactores, con un alto componente de desarrollo de una imagen corporativa de la entidad sin ánimo de lucro, en donde sea claro para los dos segmentos de clientes qué pretende la entidad promotora del cambio (OSL) y cómo actúa cada uno de ellos en beneficio de la causa.

En el mercadeo social se hace un fuerte énfasis en el desarrollo de programas de relaciones públicas y promoción, básicamente tratando de comunicar la causa a múltiples entidades interesadas o involucradas en la problemática; por eso es tan común el contacto de estas organizaciones con entidades del Estado y con medios de comunicación. Muchas campañas de mercadeo social son llevadas a los medios en forma de publicidad masiva y free press, casi siempre combinando los recursos propios con donaciones y patrocinios.

La vinculación de las empresas privadas a los programas de mercadeo social normalmente no involucra fines comerciales; esta es la principal diferencia con el mercadeo de causas o mercadeo relacionado con causas (MRC).

\section{Mercadeo de causas (MRC)}

El mercadeo relacionado con causas busca integrar la filosofía de la responsabilidad social corporativa y la filantropía . Es una sociedad a largo plazo entre un grupo sin ánimo de lucro (OSL) y una empresa, distinta a la filantropía corporativa ${ }^{8}$, que hace parte de un programa de mercadeo. La diferencia de este tipo de programas de mercadeo frente al mercadeo social ${ }^{9}$ convencional es la participación coordinada de empresa-entidad sin ánimo de lucro en una actividad planeada como estrategia de mercadeo de un producto o una marca en beneficio de una causa social.

De la definición es importante resaltar que la estrategia de mercadeo de la entidad sin ánimo de lucro consiste en crear el espacio para vincular la causa con una marca, definir la mejor forma de realizar la estrategia promocional y de patrocinio y apoyar la estrategia de mercadeo de la causa que desarrolla la empresa patrocinadora.

La empresa privada por su parte, al involucrarse una marca en una estrategia de mercadeo relacionado con la causa, debe evitar que la estrategia sea de corto plazo. Si el compromiso con la causa no está relacionado con el corazón de la empresa o de la marca, lo más probable es que se pierda credibilidad de los consumidores. La estrategia debe prevenir este problema seleccionando muy bien la causa con la que se relaciona y con el socio estratégico; es decir, la entidad sin ánimo de lucro que va a recibir el apoyo.

Este modelo, al tener fines comerciales, tiene fuertes implicaciones para la entidad sin ánimo de lucro y para la empresa patrocinadora, en este caso la relación entre las dos es más la de una alianza estratégica cuyo objetivo debe ser la búsqueda de la sinergia. El plan de mercadeo involucra a las dos entidades, así como los

6 Íbíd. 4.

7 Ídem.

8 SMITH, Scott y ALCORN, David. "Cause Marketing: A new direction in the marketing of corporate responsability". The journal of consumer marketing. Vol. 8, No. 3.

9 HOGGINS, Kevin. "Marketing with conscience". Marketing Management. Vol. 11, No. 4, 2002 
resultados del mismo y la función de mercadeo de la OSL cambia.

Para el desarrollo de la estrategia del mercadeo normalmente se apela a una de las siguientes tres formas de patrocinio 10 :

1. Apoyo para pauta o compra de espacios en medios: Una forma común de realizar este tipo de patrocinios es la compra de tiempo en los medios, donde la causa es patrocinada por la empresa y se liga a un producto. Un ejemplo de este tipo de patrocinio es el de las teletones.

2. Publicidad en medios y donaciones condicionales: En este patrocinio se incluyen actividades promocionales en las que se publicita la causa para generar una respuesta de compra en el consumidor a partir de la que se entregan donaciones a la causa. Las donaciones en dinero a la causa son proporcionales a la compra del producto o a la suscripción de un servicio. Un ejemplo de este tipo de patrocinio es el de American Express, que dona 50 centavos de dólar a las bibliotecas públicas de Florida por cada transacción realizada con su tarjeta. Esta misma empresa realizó una campaña de tres meses donando 25 centavos para el arreglo de la Estatua de la Libertad en Nueva York. El consumo de la tarjeta fue tal que donó 1.7 millones de dólares para esa causa.

3. Pauta en medios y dos o más combinaciones de incentivos para donaciones: Para este tipo de patrocinio se emplean dos o más formas de promoción para dar soporte a la causa. Una de ellas es a través de cupones que se cambian por dinero y que se ofrecen al consumidor para hacer sus donaciones a la causa. A esta forma de promoción se le pueden aplicar variantes como la que emplea $\mathrm{Al}-$ macenes Éxito, preguntando al comprador si quiere donar un pequeño excedente en su pago para una causa. Otra forma de soporte es que al comprar un producto o dar una donación por parte del consumidor, la empresa patrocinadora da a su vez su propia donación. De esta forma la causa recibe dos o más fuentes de ingreso.

Las alternativas para las actividades promocionales en el mercadeo de causas deben estar respaldadas por la convicción de la empresa patrocinadora y de sus empleados. En la medida en que estas actividades promocionales están acompañadas de una motivación de los empleados por la causa y por la promoción, los resultados en el mercado son más compatibles con el objetivo de la estrategia ${ }^{11}$.

Este tipo de estrategias de mercadeo no están exentas de críticas. Se critica, por ejemplo, la manipulación al consumidor porque se asocia un producto a la causa y esto genera una mayor venta del producto, siendo el mayor beneficiado el patrocinador mas que la misma causa. En otros casos, se asume que el efecto en la causa no es de largo plazo porque las actividades promocionales pueden ser de cortos lapsos de tiempo. Hay que mencionar que aunque el debate está abierto, se debe considerar que el mercadeo de causas es una evolución del mercadeo social tradicional que ha mostrado ser eficaz para obtener resultados a favor de una causa, pero que no logra generar el interés suficiente de las empresas y muchas veces queda corto para la consecución de fondos para los programas que requieren desarrollarse a favor de la causa. Mediante el mercadeo de causas se logra una masificación de la causa con una respuesta voluntaria del consumidor, lo cual genera movimientos sociales a favor de la causa además del beneficio económico.

Uno de los problemas que se mencionan al realizar mercadeo de causas es el rechazo del consumidor porque se siente manipulado. En estudios sobre el mercadeo de causas en consumidores, se ha mostrado todo lo contrario; la opinión es que para ellos es mucho mejor comprarle a una compañía que se sabe que hace algo positivo por la comunidad 12 .

Se ha encontrado que los consumidores prefieren hacer una donación o comprar un producto a empresas relacionadas con causas, porque para ellos se genera un diferencial si los productos, precios, o posicionamiento frente a empresas competidoras es semejante ${ }^{13}$. También se ha encontrado que no todos los consumidores responden a este tipo de mercadeo, sino aquellos que no han tomado una decisión por una marca o que hacen compras ocasionales de la misma ${ }^{14}$, y además, que quienes se adhieren a este tipo de promociones son consumidores que, de alguna forma, ven un beneficio

10 Íbíd. 2.

11 Íbíd. 3.

12 MEYER, Harvey. "When the cause is just". The journal of Business Strategy. Vol. 20, NO. 6, 1999.

13 BARONE, Michael, MIYAZAKI, Anthony y TAYLOR, Kimberly. "The influence of cause-marketing on consumer choice: Does one good turn deserve another?", Academy of marketing Science Journal. Vol. 28, NO. 2, 2000.

14 VAN DEN BRINK, Douwe, ODEKERKEN-SCHRÖDER, Gaby y PAWELS, Pieter. "The effect of strategic and tactical cause-related marketing on consumer's brand loyalty". The journal of consumer marketing. Vol. 23, NO. 1, 2006 
próximo de la causa; por ejemplo, vinculado al desarrollo local ${ }^{15}$, muchos consumidores solo apoyan causas con las que se identifican por múltiples razones personales, más que por la sola existencia de la promoción.

El mayor reto al asumir este tipo de programas es el logro de la sinergia entre las expectativas de la empresa que desarrolla el mercadeo de la causa y la entidad sin ánimo de lucro que la promueve. En ese sentido, Meyer ${ }^{16}$ sugiere considerar los siguientes puntos en el diseño de la estrategia:

1. Sinergia entre el programa de la causa y los objetivos estratégicos de negocio de la empresa. 2. Compromiso de los directivos de más alto rango de la empresa.

3. Suficiencia de recursos para el desarrollo de las campañas y el pago de los espacios en los medios.

4. Compromisos a lago plazo.

5. Relación abierta y mutuamente benéfica.

6. Creación de múltiples mecanismos de comunicación con el público.

7. Resultados medibles.

8. Mercadeo interno de la causa en los empleados.

9. Innovación o dinamismo en la forma de abordar la causa y mantener interesado al consumidor.

El mercadeo de causas implica afrontar otro tipo de retos para la entidad sin ánimo de lucro, que a la vez va a llevar a resultados que se alejan de la filantropía y del mercadeo social tradicional.

\section{Barreras para el mercadeo de causas ambientales}

Como se mostró anteriormente, el mercadeo de causas ambientales tiene múltiples alternativas; sin embargo, al inclinarse por una u otra o al combinarlas, la gestión del mercadeo trae consigo enfrentarse a una serie de barreras o puntos críticos sobre los cuales se debe actuar para obtener el mejor resultado posible para la entidad y sobre todo, para la causa misma. Estas barreras se pueden resumir en las siguientes:

- Relacionadas con la comunicación organizacional y el mercadeo interno en la OSL y la educación de los aliados empresariales.
- Relacionadas con la imagen de la entidad sin ánimo de lucro.

- Relacionadas con los procesos de negociación con las empresas privadas.

- Relacionadas con la fidelidad de los benefactores, donantes o patrocinadores.

- Relacionados con el impacto del mercadeo y el impacto social.

En los dos primeros casos, la construcción de una imagen institucional y la comunicación interna y con los públicos externos implican un trabajo coordinado de mercadeo con las oficinas de prensa y comunicación de la entidad sin ánimo de lucro y de éstas con los medios, las entidades estatales y las oficinas de las empresas benefactoras, donantes o patrocinadoras.

La labor de mercadeo se puede ver afectada principalmente por la credibilidad de la entidad sin ánimo de lucro, de las acciones que realiza o de los programas específicos que desarrolla o haya desarrollado en el pasado.

La credibilidad del público en general y de las empresas en particular, en la entidad sin ánimo de lucro, está mediada por la imagen que ésta tenga en los distintos medios (empresarial, social o incluso estatal) y por el comportamiento que se observe en todos sus empleados y actos públicos.

El punto crítico, en este caso, es lograr transmitir una imagen coherente a través de las diferentes actividades de cada área, entendiendo que la imagen corporativa de la entidad sin ánimo de lucro es "la experiencia global con la empresa", como señala Van Riel ${ }^{17}$ y planear la comunicación de la entidad y la imagen que se desea proyectar siguiendo un proceso orientado como el que propone Sanz de la Tajada ${ }^{18}$, que pasa por el análisis de la situación, la construcción de la identidad o ideograma de la ESL, la identificación de públicos diferenciados, los atributos que se quieren proyectar, el posicionamiento estratégico y desde allí, la estrategia de medios y de mensajes y los planes de acción por ejecutar.

15 OSTERHUS Thomas. "Pro-social consumer influence strategies: When and how do they work?" Journal of marketing. Vol. 61, No. 4,

1997

16 Íbíd. 12.

17 VAN RIEL, Cees (1997). Comunicación Corporativa. Madrid: Editorial Prentice Hall.

18 SANZ DE LA TAJADA, Luis (1994). Integración de la identidad y la imagen de la empresa. Madrid: Editorial ESIC. 
En cuanto a los procesos de negociación con las empresas privadas, realmente éstos se convierten en el proceso de gestión comercial que realiza el departamento de mercadeo de la entidad sin ánimo de lucro con las empresas privadas que se pueden convertir en potenciales benefactoras o patrocinadoras. La negociación consistirá en llegar a un acuerdo sobre los objetivos de ambas partes y sobre los beneficios que obtendrá cada una en la relación que se entabla.

El proceso de negociación en estos casos es complejo por la multiplicidad de objetivos e intereses de cada actor y por las implicaciones económicas y estratégicas que tiene para los participantes. Se debe tener en cuenta en este punto que el hecho de que la causa sea benéfica para la sociedad o para una necesidad, a la empresa no le asegura una disposición a negociar; para que ésta sea posible se deben entender los procesos de decisión en la empresa benefactora, las personas que intervienen, los roles de cada individuo y las objeciones posibles a la propuesta de la ESL.

El punto crítico en este caso es mostrar la convergencia de intereses y beneficios para los dos actores y hacerlo a través de una técnica de negociación bien planeada. Dentro de la planeación de la negociación se debe considerar que seguramente participarán grupos de personas de ambas organizaciones y que el proceso no es rápido por ser asimilable a un proceso de compra institucional sin experiencia previa.

De otra parte, hay que tener la suficiente flexibilidad para plantear alternativas y de esta forma, obtener buenos resultados en la negociación; como sugieren De Manuel y Martínez-Vilanova ${ }^{19}$, el proceso de negociación deberá incluir al menos cuatro fases: presentación de propuestas, establecimiento de límites y agenda de negociación, negociación y alternativas y, conclusión y acuerdo.

Respecto a la fidelización, una barrera importante para el mercadeo de las causas ambientales es poder mantener una base de benefactores, donantes o patrocinadores estables, con quienes se puedan recibir apoyos o trabajar conjuntamente múltiples iniciativas y programas. La fidelidad se puede lograr desarrollando programas específicos dirigidos a las empresas o a las personas que dentro de ellas apoyan la causa o la defienden, y acercando a aquellos que pueden convertirse en detractores.

Para construir un plan de fidelización es necesario tener en cuenta que, ante todo, se deben manejar muy bien las relaciones con estas empresas, no solo en el sentido de servicio al cliente, sino en el sentido de comprensión del cliente, máxime cuando las empresas benefactoras buscan obtener resultados estratégicos a partir de la relación con la entidad sin ánimo de lucro. Para poder comprender al benefactor es necesario establecer claramente cuáles son los beneficios reales que éste percibe, cuáles los riesgos a que se ve expuesto o que percibe de manera errónea, cuáles los costos en que incurre y cuáles las posibilidades que pueden presentársele. En este sentido, la fidelización corresponde a un programa específico dentro de la estrategia de mercadeo relacional de la entidad sin ánimo de lucro.

Los puntos críticos en este caso son: comprender, crear vínculos, facilitar los procesos y detectar los cambios.

Sobre la creación de vínculos, Alet ${ }^{20}$ sugiere tener presente la evolución de la relación a partir de una mirada en escalones donde se va avanzando en los niveles de lealtad desde el cliente potencial hasta el socio. La creación de las ofertas se adaptará a esos niveles de lealtad y de esta manera, se podrán ir creando vínculos financieros, sociales y estructurales (aquellos en donde la entidad sin ánimo de lucro y la empresa benefactora adaptan sus sistemas o su organización para desarrollar la relación). La facilitación de procesos se va dando en la medida en que se construye el vínculo. La detección de cambios implica un monitoreo permanente de la satisfacción y el valor percibido por la empresa benefactora, tratando de comprender los costes en que se incurren y las presiones del medio que puedan presentar. El análisis permanente del entorno en el que se mueve la empresa benefactora facilita la comprensión de los cambios a que está sometida y permite desarrollar opciones estratégicas para evitar el abandono.

Respecto a la barrera que constituye el impacto del mercadeo y el impacto social, hay que considerar que el impacto del mercadeo puede estar sujeto al impacto social; es decir, la participación de las empresas puede estar condicionada a la credibilidad de la ESL, a su pro-

19 DE MANUEL, Fernando y MARTÍNEZ - VILANOVA, Rafael (2000). Comunicación y negociación comercial. Madrid: Editorial ESIC. 20 ALET, Joseph (2001). Marketing relacional. Barcelona: Editorial Gestión. 
pio impacto en la sociedad o al impacto de la estrategia que se construye conjuntamente. Desde este punto de vista, para mejorar el impacto éste debe ser medido y convertido en una prioridad para la entidad sin ánimo de lucro. El problema se presenta cuando hay múltiples objetivos, públicos y responsables del impacto. Una de las mayores dificultades para poder conocer el impacto es generar sistemas de medición de los mismos, en los que sea posible separar los resultados correspondientes a cada programa y entidad, de los resultados conjuntos.

El punto crítico en este caso es definir sistemas de indicadores de impacto basados en varias fuentes. No solo considerar el resultado económico, sino también el posicionamiento, los cambios en la opinión pública, los resultados en la adopción de la causa que sean demostrables y la medición de los efectos inmediatos y de corto plazo de las campañas. Este es, tal vez, uno de los puntos más complejos de tratar en la estrategia de mercadeo.

A modo de conclusión, quisiera señalar que mercadear causas ambientales requiere un alto nivel de formalización y planeación, así como creatividad; esto dependerá del manejo concienzudo por parte de las entidades sin ánimo de lucro para que las empresas y todos nosotros podamos involucrarnos de una $\mathrm{u}$ otra forma y se puedan obtener los resultados que requiere la humanidad.

\section{BIBLIOGRAFÍA}

- WYMER, Walter. "Special issue on corporate philanthropy". En International Journal of Nonprofit and Voluntary Sector Marketing. Vol. 11, No. 1, 2006.

- HOGGINS, Kevin. "Marketing with conscience". En Marketing Management. Vol. 11, No. 4, 2002.

- DAELLENBACH, Kate, DAVIES, Jhon y ASHILL, Nicholas. "Understanding sponsorship relationships-multiple frames and multiple perspectives". En International Journal of Nonprofit and Voluntary Sector Marketing. Vol. 11, No. 1, 2006.

- FOX, Karen y KOTLER, Philip. "The marketing of social causes: The first 10 years". En Journal of Marketing. Vol. 44, No. 4, 1980 .

- SMITH, Scott \& ALCORN, David."Cause Marketing: A new direction in the marketing of corporate responsability". En The journal of Consumer Marketing. Vol. 8, No. 3.

- MEYER, Harvey. 'When the cause is just". En The journal of Business Strategy. Vol. 20, No. 6, 1999.

- BARONE, Michael, MIYAZAKI, Anthony y TAYLOR, Kimberly. "The influence of cause-marketing on consumer choice: Does one good turn deserve another?". En Academy of Marketing Science Jourrnal. Vol. 28, No. 2, 2000.

- VAN DEN BRINK, Douwe, ODEKERKEN-SCHÖDER, Gaby y PAWELS, Pieter. "The effect of strategic and tactical cause-related marketing on consumer's brand loyalty". En The journal of Consumer Marketing. Vol. 23, No. 1, 2006.

- OSTERHUS, Thomas. "Pro-social consumer influence strategies: When and how do they work?" En Journal of Marketing. Vol. 61, No. 4, 1997.

- VAN RIEL, Cees (1997). Comunicación Corporativa. Madrid: Editorial Prentice Hall.

- SANZ DE LA TAJADA, Luis (1994). Integración de la identidad y la imagen de la empresa. Madrid: Editorial ESIC.

- DE MANUEL, Fernando y MARTÍNEZ-VILANOVA, Rafael (2000). Comunicación y negociación comercial. Madrid: Editorial ESIC.

- ALET, Joseph (2001). Marketing relacional. Barcelona: Editorial Gestión. 